$\begin{array}{ll}\text { Research Square } & \text { Preprints are preliminary reports that have not undergone peer review. } \\ \text { They should not be considered conclusive, used to inform clinical practice, } \\ \text { or referenced by the media as validated information. }\end{array}$

\title{
Association between aerobic exercise and handgrip strength in adults: a cross-sectional study based on data from the Korean National Health and Nutrition Examination Survey (2014-2017)
}

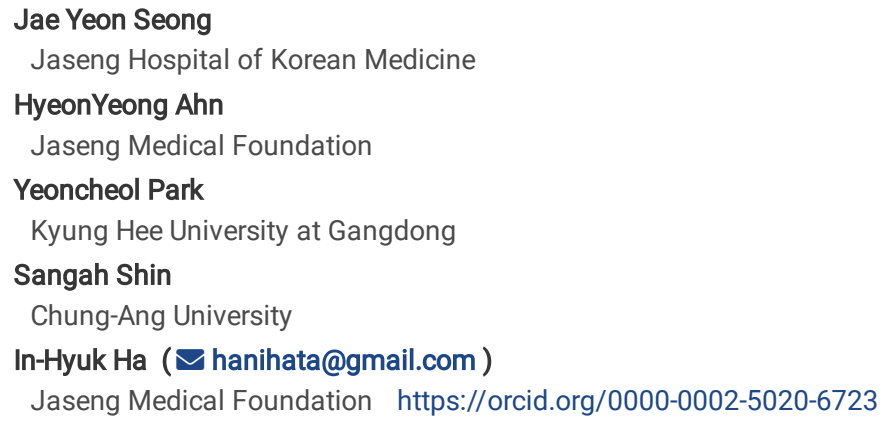

Keywords: Aerobic exercise, Cross-sectional study, Diabetes mellitus, Handgrip strength, Hypertension, Korean National Health and Nutrition Examination Survey

Posted Date: November 13th, 2019

DOI: https://doi.org/10.21203/rs.2.17177/v1

License: (c) (1) This work is licensed under a Creative Commons Attribution 4.0 International License. Read Full License

Version of Record: A version of this preprint was published at The journal of nutrition, health \& aging on April 25th, 2020. See the published version at https://doi.org/10.1007/s12603-020-1372-x. 


\section{Abstract}

Background Handgrip strength is an easy-to-assess indicator of overall muscle strength and can be used to evaluate health status. Although previous studies have reported an increase in grip strength due to aerobic exercise, such a study has not been conducted on Korean participants. This study aimed to investigate the effects of aerobic exercise on handgrip strength and examine the association between these two variables in Korean patients with hypertension or diabetes.

Methods This cross-sectional study used data from the 6th and 7th Korean National Health and Nutrition Examination Survey (2014-2017). A total of 19,650 individuals aged $\geq 19$ years who had responded to questionnaires concerning aerobic exercise and handgrip strength were analyzed. The relationship between aerobic activity and handgrip strength was examined by logistic regression analysis.

Results The mean age of individuals in the low muscle strength group was higher than that in the normal muscle strength group. The odds ratio for low handgrip strength was higher in individuals who did not perform aerobic exercise than in those who performed aerobic exercise. Following adjustment for covariates, the odds ratios ( $95 \%$ confidence intervals) for low handgrip strength were $1.415(0.187-1.688)$ in the total sample, 1.799 (1.376-2.352) in patients with hypertension, and $1.811(1.208-2.715)$ in patients with diabetes.

Conclusion The results of our study indicated a strong association between aerobic exercise and handgrip strength in the Korean population.

\section{Background}

Handgrip strength, which refers to the force used to grasp an object with the hand, is a simple, rapid, and standard indicator for assessing overall muscle strength [1]. Additionally, it is an efficient technique for evaluating the overall health condition, including nutritional status and gait [2]. Recent studies have reported handgrip strength as related to hypertension [3], diabetes [4], and other various chronic diseases [5], as well as to quality of life [6] and mental health, including depression [7] and dementia [8].

Physical activity refers to all bodily movements that consume energy for skeletal muscle activity [9] and can be broadly divided into aerobic and anaerobic exercises. Aerobic exercise refers to the physical activity that utilizes carbohydrates and lipids in the body as the energy source, which increases oxygen consumption for a certain duration for the combustion and oxidation of carbohydrates and lipids [10]. This form of exercise is effective in preventing and correcting obesity by reducing body fat [11] and has positive effects on chronic diseases, including hypertension [12], cardiovascular disease [13], and diabetes [14]. Therefore, public health institutions from various countries recommend aerobic exercise to support the prevention and treatment of chronic diseases [15]. Aerobic exercise also has positive effects on mental health disorders, such as depression [16] and anxiety [17], and can improve the overall quality of life [18]. Thus, regular aerobic exercise is essential for maintaining and improving both physical and mental health.

Physical activity recommendations for the residents of various countries, including Japan, South Korea, and North American countries, have been published. In 2013, the Ministry of Health and Welfare had published the "Physical Activity Guidelines for South Koreans" as recommendation and legislation of the standards of physical activity for the improvement in health of the national population [9]. However, according to a report published by the Ministry of Health and Welfare, only 3 out of 10 adults in South Korea adhered to these guidelines [19]. Additionally, the 2017 Korean National Health and Nutrition Examination Survey (KNHANES) showed a rapid decrease in the percentage of the population performing aerobic exercise, from $58.3 \%$ in 2014 to $48.4 \%$ in 2017 [20].

Although aerobic exercise generally improves aerobic capacity and is associated with cardiovascular function and metabolic control [21], it has minimal effects on the improvement in musculoskeletal mass and function, for which muscle-strengthening exercise is regarded as more suitable. However, numerous recent studies have reported the association between aerobic exercise and muscle strength. In a study by Pikosky et al. [22], adults who participated in a 4week aerobic exercise program showed significantly increased skeletal muscle fractional synthetic rate. In a literature review by Konopa and Harber [21], aerobic exercise was reported to increase muscle fiber and overall muscle size via mechanisms involving modulation of the skeletal muscle molecules and protein metabolism.

In South Korea, diabetes is responsible for 10,000 deaths annually, making it the $6^{\text {th }}$ leading cause of death [23]. In 2016, the prevalence of diabetes increased to $11.3 \%$ from $9.5 \%$ in 2015 [20]. Hypertension is the most common and strongest risk factor associated with progression to cardiovascular disease, and its prevalence has shown a gradual increasing trend from $27.8 \%$ in 2015 to $29.1 \%$ in 2016 [24]. These are two of the three major chronic diseases among Koreans [20], which are difficult to cure, require long-term management, and can result in permanent disability [25].

Among previous studies on handgrip strength and aerobic exercise, Crane et al. [26] showed that long-term aerobic exercise improved cardiopulmonary and metabolic functions and alleviated age-related loss of muscle strength. Al-Sharif et al. [27] implemented treadmill-based aerobic exercise in patients with hemophilia and reported significant increases in serum calcium concentration and handgrip strength. In a study by Al-Shreef et al. [28], aerobic exercise significantly increased bone metabolism and handgrip strength in patients with type 2 diabetes. Mital et al. [29] directed aerobic exercise in patients with coronary artery disease and observed a significant increase in handgrip strength.

However, the previous studies have all used relatively small study populations; additionally, there has not yet been any study on the relationship between handgrip strength and aerobic exercise in the Korean population. In this study, we used data from the 2014-2017 KNHANES to examine changes in handgrip strength based on aerobic exercise behaviors in Korean adults aged $\geq 19$ years and to investigate this relationship in patients with hypertension and those with diabetes.

\section{Methods}


- Study population

This study used raw data from KNHANES-VI and -VII (2014-2017). KNHANES is a nationwide cross-sectional survey that has been conducted since 1988 to ascertain the state of health and nutrition in the South Korean population, in accordance with Article 16 of the National Health Promotion Act, by the Korean Centers for Disease Control and Prevention (KCDC), as part of the Ministry of Health and Welfare. A representative sample of the households is selected by systematic stratified cluster sampling, and household members $\geq 1$ years of age, excluding institutionalized individuals, are subjected to a health questionnaire, health examination, and nutritional survey. In the present study, out of 31,207 total respondents in the survey, we excluded those aged $<19$ years (6,386 individuals) as well as those with missing values for handgrip strength (3,667 individuals) and exercise data (1,504 individuals), and we included the remaining 19,650 respondents (8,676 men, 10,974 women) in our analysis (Fig. 1).

- Handgrip strength

From visual inspection and interview with the participants, the surveyors excluded those in whom measuring handgrip strength was difficult due to functional limitations, history of hand or wrist surgery within the last 3 months, or hand or wrist pain within the last week. For the measurement of handgrip strength, participants adopted a standing position with their lower arms hanging naturally, away from the body, at the height of their thigh, and grasped the dynamometer without bending their elbow or wrist. Measurements were taken for a duration of up to 3 seconds. Three measurements were taken from each hand, alternatively, for a total of 6 measurements. The maximum value from the dominant hand was used as the final measurement of grip strength. The device used for measurement was a digital grip strength dynamometer (TKK 5401; Takei Scientific Instruments Co, Ltd, Tokyo, Japan). In a previous study using KNHANES data by Kim et al. [30], cut-off values for handgrip strength were defined to be $<28.9 \mathrm{~kg}$ for men and $<16.8 \mathrm{~kg}$ for women, and applying the same values in our study, we classified individuals with handgrip strength below the cut-off value as "low muscle strength" (LMS) and those with handgrip strength above the cut-off values as "normal muscle strength" (NMS).

\section{- Aerobic exercise}

Aerobic exercise performance rate was defined as the fraction of participants who performed, per week, at least $2 \mathrm{~h}$ and 30 min of moderate physical activity, 1 $\mathrm{h}$ and $15 \mathrm{~min}$ of vigorous physical activity, or an equivalent mixture of moderate and vigorous physical activity (where 1 min of vigorous activity is equivalent to 2 min of moderate activity). Using questionnaire items on aerobic exercise, we first identified participants who responded "Yes" to the question "Does your day include at least $10 \mathrm{~min}$ of vigorous/moderate physical activity, during which you are continuously very/slightly out of breath or your heart beats much/slightly faster?," or to the question "Do you usually perform at least $10 \mathrm{~min}$ of vigorous/moderate sports, exercise, or leisure activities, during which you are continuously very/slightly out of breath or your heart beats much/slightly faster?"; then, based on the responses of these participants to the question "In a typical week, how many days/hours due to perform vigorous/moderate physical activity related to work/leisure?," we calculated the number of people who performed at least $2 \mathrm{~h}$ and $30 \mathrm{~min}$ of moderate physical activity, $1 \mathrm{~h}$ and $15 \mathrm{~min}$ of vigorous physical activity, or an equivalent mixture of moderate and vigorous physical activity per week. The measurement results for vigorous/moderate physical activity were converted into minutes, and when the amount of exercise was calculated in terms of metabolic equivalent (MET-min/week, hereafter MET), participants were classified into aerobic exercisers ( $\geq 600$ MET$\mathrm{min} /$ week) and non-exercisers (<600 MET-min/week).

-

- Covariates

As sociodemographic characteristics of the participants, we included age, sex, educational level, marital status, and income level. Similarly, as lifestyle habit factors, we included weight, height, smoking status, and heavy alcohol drinking; as disease- and health-related factors, we included diagnosis of hypertension and diabetes, body mass index (BMI), and forced expiratory volume in 1 second ( $\left(\mathrm{FEV}_{1}\right)$. Educational level was categorized into elementary school graduation or below, middle school graduation, high school graduation, and college graduation or above; income level was categorized into 4 quartiles: low, lower-middle, upper-middle, and high. BMI was calculated as the weight in kilograms divided by the square of the height in meters. Smoking status was categorized as never smoker, past smoker, or current smoker. Heavy alcohol drinking was calculated as the number of participants (n) who responded that they drank on an average $\geq 7$ servings at a time for men or $\geq 5$ servings at a time for women at least twice per week. Hypertension prevalence was calculated as the number of participants ( $\mathrm{n}$ ) with systolic blood pressure $\geq 140 \mathrm{mmHg}$, diastolic blood pressure $\geq 90 \mathrm{mmHg}$, or who reported as taking antihypertensive medication. Diabetes prevalence was calculated as the number of participants ( $\mathrm{n}$ ) with fasting blood glucose $\geq 126 \mathrm{mg} / \mathrm{dL}$, diagnosed with diabetes by a doctor, reported as taking blood glucose lowering agents or insulin treatments. $\mathrm{FEV}_{1}$ was measured in patients aged $\geq 40$ years using dry rolling-seal spirometry (Vmax series 2130; SensorMedics, USA).

- Statistical methods

As KNHANES is a sample survey rather than a census, all data analyses were performed using a weighted complex sample design. For participants' sociodemographic data, measurements, and examination results, categorical variables were expressed as frequency and percentage (\%), whereas continuous variables were presented as mean and standard deviation. Categorical and continuous variables were analyzed using chi-square test and t-test, respectively, and $p<0.05$ was considered significant. In order to analyze the effects of aerobic exercise on handgrip strength, we performed a logistic regression analysis and obtained the odds ratios (ORs) and confidence intervals. SPSS version 25.0 (IBM Corp., Armonk, NY, USA) was used for all statistical analyses in this study.

- Ethics statement

KNHANES-VI and -VII were conducted by the KCDC, and all participants provided prior written consent. All survey protocols were approved by the institutional review board of the KCDC (approval numbers 2013-12EXP-03-5C and 2015-01-02-6C). Data from KNHANES are available on the "Korea National Health

Page 3/10 
and Nutrition Examination Survey" website (http://knhanes.cdc.go.kr), specifically the needed annual reports. Our study was approved by an institutional review board (JASENG 2019-04-006).

\section{Results}

A total of 19,650 individuals were selected as participants in this study, including 8,676 men and 10,974 women. To analyze the general characteristics, participants were divided into the LMS and NMS groups, both for the entire study population and for men and women separately. Across all participants, the mean age of participants in the LMS group was $65.0 \pm 16.5$ years, whereas that of participants in the NMS group was $48.6 \pm 15.7$ years, indicating that patients in the LMS group were older on average, and these trends were similar when the analysis was repeated separately for men and women (men: LMS, $66.2 \pm 16.1$ years; NMS, $48.7 \pm 15.8$ years and women: LMS, $64.0 \pm 16.8$ years; NMS, $48.5 \pm 15.7$ years). Across all participants, the mean grip strength of the dominant hand was $18.1 \pm 6.1 \mathrm{~kg}$ in the LMS group and $31.2 \pm 9.6 \mathrm{~kg}$ in the NMS group, indicating that the NMS group showed stronger handgrip (men: LMS, $24.7 \pm 5.1 \mathrm{~kg} ; \mathrm{NMS}$, $38.3 \pm 6.5 \mathrm{~kg}$ and women: LMS, $13.9 \pm 3.0 \mathrm{~kg}$; NMS, $22.7 \pm 4.0 \mathrm{~kg}$ ). Based on lifestyle habits, a total of $788(32.0 \%)$ and $8,434(49.1 \%)$ individuals performed aerobic exercise in the LMS and NMS groups, respectively; excluding the male NMS group $(4,016$ individuals [52.7\%]), there were more non-exercisers than exercisers in all groups. Among health-related factors, across all participants, there were 1,194 (48.5\%) and 4,865 (28.4\%) patients with hypertension in the LMS and NMS groups, respectively; furthermore, there were 475 (22.3\%) and 1,671 (10.3\%) patients with diabetes in the LMS and NMS groups, respectively (Table 1).

We performed logistic regression analysis to examine the changes in handgrip strength with respect to aerobic exercise status (Table 2). Model 1 was adjusted for covariates; Model 2 was adjusted for age, sex, and BMI, which could affect handgrip strength; Model 3 was Model 2 adjusted for all other sociodemographic factors, lifestyle habit factors, and disease- and health-related factors. In the separate analyses of men and women, the adjustment for sex was omitted. In the univariate analysis of all participants, the OR was significant, at 2.053 (1.867-2.245), and this association was significant even for individual analysis of men and women using the same model [men: 1.968 (1.723-2.249), women: 2.123 (1.879-2.399)]. Models 2 and 3, adjusted for covariates, also showed significant ORs in all cases, at 1.448 (1.315-1.594) and 1.415 (1.187-1.688), respectively, across all participants: 1.451 (1.256$1.676)$ and $1.511(1.162-1.965)$ in men and $1.446(1.269-1.647)$ and $1.372(1.079-1.745)$ in women $(p<0.05)$.

Table 2 presents the results of analysis of the relationship between aerobic activity and handgrip strength in patients with hypertension, which shows the highest prevalence of all chronic diseases in South Koreans. Additionally, among patients with hypertension, the ORs for low grip strength in non-exercisers compared with exercisers were significant at 2.267 (1.964-2.617) in Model 1, 1.610 (1.373-1.888) in Model 2, and 1.799 (1.376-2.352) in Model 3. In the analysis according to sex, both men and women showed significantly high ORs for low grip strength when not performing aerobic exercise; particularly, when all confounding variables were adjusted for, we observed a slightly stronger association in men than in women [men: 1.910 (1.306-2.794), women: 1.796 $(1.218-2.648)]$.

Table 2 also shows the results of analysis of the relationship between aerobic exercise and handgrip strength in patients with diabetes. In the crude model including all participants, non-exercisers showed a significantly increased OR for LMS (2.344 [1.858-2.957]) when compared with exercisers, and the results were also consistent in Models 2 and 3 (Model 2, 1.793 [1.396-2.303]; Model 3, 1.811 [1.208-2.715]). In the analysis according to sex, men showed even stronger associations in the models corrected for covariates (Model 2, 1.679 [1.201-2.348]; Model 3, 2.032 [1.155-3.574]) compared with the crude model (1.958 [1.437-2.667]). When not performing aerobic exercise, the ORs for LMS in women were significant in Model 1 at 2.787 (1.938-4.009) and in Model 2 at 2.001 (1.364-2.937).

\section{Discussion}

In this study, we analyzed the relationship between aerobic activity and handgrip strength based on reliable, large-scale nationwide data.

Using data from KNHANES-VI and -VII (2014-2017), out of 31,207 total participants, we analyzed 19,650 individuals (8,676 men, 10,974 women) aged $\geq 19$ years who had available data on both grip strength and aerobic exercise. The results clearly indicated a higher risk of low grip strength in South Koreans aged $\geq 19$ years who did not perform aerobic exercise. We also performed subgroup analyses of participants with hypertension or diabetes, which are two of the major chronic diseases in South Korea. Among patients with hypertension or diabetes, individuals who did not perform aerobic exercise showed significantly increased risk of low grip strength.

The mechanism for reduced handgrip strength in individuals performing less aerobic exercise can be explained by the theory of muscle protein balance due to increased mitochondria [21, 22]. Lack of aerobic exercise increases levels of inflammatory factors such as cytokines and tumor necrosis factor-alpha (TNF-a), and this leads to reduced muscle strength [31]. Because inflammation is inversely proportional to insulin-like growth factor-1 (IGF-1) levels, reduced IGF-1 leads to a decrease in the muscle mass, and the inflammatory factor TNF-a induces cell death in muscles, leading to impaired muscle function [32]. During aerobic exercise, mitochondrial count and activity increase, balancing muscle proteins by controlling muscle protein degradation and synthesis, and promoting growth of skeletal muscle [21,22]. In addition, lack of aerobic exercise leads to increased lipid mass, and this lipid accumulates inside muscle fibers, leading to impaired skeletal muscle function [33].

In the present study, we performed a subgroup analysis to investigate the relationship between aerobic exercise and handgrip strength in patients with hypertension or diabetes, both of which have a high prevalence in South Koreans. In both patients with hypertension and diabetes, less aerobic exercise was associated with a significant increase in the risk of low handgrip strength. Previously, very few studies have examined the relationship between handgrip strength and aerobic exercise in patients with diabetes and hypertension. 
In an animal study by Ergen et al. [34], when diabetes-induced rats performed 8 weeks of treadmill exercise, they showed significantly lower blood glucose and increased skeletal muscle endurance capacity compared to rats who did not perform aerobic exercise. According to the results of a study by Wallymahmed et al. [35], when aerobic capacity $\left(\mathrm{VO}_{2} \mathrm{max}\right)$, handgrip strength, and hemoglobin $\mathrm{A} 1 \mathrm{c}$ were measured in patients with type 1 diabetes, individuals with high $\mathrm{VO}{ }_{2}$ max exhibited higher handgrip strength, but showed a trend for worse blood glucose control. Al-Shreef et al. [28] implemented 6-month aerobic exercise or resistance exercise in 100 patients with type 2 diabetes and observed significant increases in bone metabolism and grip strength in both the aerobic exercise group and resistance exercise group. In addition to its main role in physical activity, skeletal muscle is also involved in insulin metabolism and controls blood glucose via insulin-mediated glucose uptake. When this function declines, because insulin resistance can lead to type 2 diabetes, skeletal muscle growth and elevated insulin sensitivity can be explained in terms of aerobic exercise [36].

In a study by Fernandes et al. [37] in spontaneously hypertensive rats, 10 weeks of aerobic exercise was effective in reducing blood pressure and correcting capillary rarefaction in the skeletal muscles. This is because aerobic exercise normalizes capillary infarction in skeletal muscles, reduces total peripheral resistance, and promotes increased microcirculatory flow, leading to increased blood flow due to higher numbers of blood vessels in the skeletal muscles. Aerobic exercise increases the ratio of capillaries-to-muscle fibers in skeletal muscles.

Our study had several limitations. First, as a cross-sectional study, although we were able to investigate correlations between variables, we were unable to clearly examine the order of changes in order to identify causal relationships. However, because we used data from KNHANES, which is representative of the South Korean population, there is little risk of sampling error. Second, because the questionnaire on aerobic exercise was dependent on the participants' memory, it is possible that memory decay bias could have reduced the accuracy of the information. Third, because the study was based on data from a survey of South Koreans, the results could have been affected by the racial characteristics of Koreans and, thus, may not be applicable to individuals of other races. Nevertheless, the value of our research is that we demonstrated a very strong association between aerobic exercise and handgrip strength, and this study was the first to examine the relationship between these two variables in the Korean adults.

\section{Conclusions}

This study investigated the relationship between aerobic exercise and handgrip strength using survey data from South Koreans. Performing less aerobic exercise was associated with higher risk of low handgrip strength, and this relationship was also significant in patients with hypertension and/or diabetes. This study demonstrates a close relationship between aerobic exercise and handgrip strength, which is a useful measure reflective of the overall physical health. In addition to its typical benefits, aerobic exercise also has excellent effects on muscle hypertrophy and modulation of blood glucose and blood pressure. Our results provide evidence that emphasizes the necessity for aerobic exercise for better health in the current population. In addition, because this study was conducted in the South Korean population, further interventional studies on the relationship between aerobic exercise and handgrip strength and the mechanisms for this relationship in other races are required.

\section{Abbreviations}

BMI: Body mass index

$F E V_{1}$ : Forced expiratory volume in 1 second

IGF-1: Insulin-like growth factor-1

$K C D C$ : Korean Centers for Disease Control and Prevention

KNHANES: Korean National Health and Nutrition Examination Survey

LMS: Low muscle strength

MET: Metabolic equivalent

NMS: Normal muscle strength

ORs: Odds ratios

TNF-a: Tumor necrosis factor-alpha

\section{Declarations}

- Ethics approval and consent to participate

- KNHANES-VI and -VII were conducted by the KCDC, and all participants provided prior written consent. All survey protocols were approved by the institutional review board of the KCDC (approval numbers: 2013-12EXP-03-5C, and 2015-01-02-6C), and our study was approved by an institutional review board (JASENG 2019-04-006).

- Consent for publication

- Not applicable 


\section{Availability of data and materials}

- The datasets generated and/or analysed during the current study are available in the "Korea National Health and Nutrition Examination Survey" website (http://knhanes.cdc.go.kr).

\section{Competing interests}

The authors declare that they have no competing interests.

- Funding

This research did not receive any specific grant from funding agencies in the public, commercial, or not-for-profit sectors.

- Authors' contributions

JYS planned the study design. JYS and HYA wrote the draft. YCP, SAS, and IHH reviewed the article. All authors read and approved the final manuscript.

- Acknowledgements

Not applicable.

\section{References}

1. Mainous AG 3rd, Tanner RJ, Anton SD, Jo A. Grip strength as a marker of hypertension and diabetes in healthy weight adults. Am J Prev Med. 2015;49:850-8.

2. Roberts HC, Denison HJ, Martin HJ, Patel HP, Syddall H, Cooper C, et al. A review of the measurement of grip strength in clinical and epidemiological studies: towards a standardized approach. Age Ageing. 2011;40:423-9.

3. Lee JA. Relationship between grip strength and prevalence of hypertension in Korean adults: The Sixth Korea National Health and Nutrition Examination Survey (2015). J Korean Acad Kinesiol. 2017;19:53-60.

4. Jang MS, Kim HK, Oh B. Comparison of hand-grip strength between normal Korean adults and those with type 2 diabetes: $2014-2015$ Korea National Health and Nutrition Examination Survey. Korean J Fam Pract. 2018;8:654-61.

5. Rantanen T, Masaki K, Foley D, Izmirlian G, White L, Guralnik JM. Grip strength changes over 27 yr in Japanese-American men. J Appl Physiol. 1998;85:2047-53.

6. Oh YH, Moon JH, Kong MH, Oh B, Kim HJ. The association between hand grip strength and health-related quality of life in Korean adults. Korean J Sports Med. 2017;35:103-11.

7. Fukumori N, Yamamoto Y, Takegami M, Yamazaki S, Onishi Y, Sekiguchi M, et al. Association between hand-grip strength and depressive symptoms: Locomotive Syndrome and Health Outcomes in Aizu Cohort Study (LOHAS). Age Ageing. 2015;44:592-8.

8. Shin HY, Kim SW, Kim JM, Shin IS, Yoon JS. Association of grip strength with dementia in a Korean older population. Int J Geriatr Psychiatry. 2012;27:5005.

9. Division of Health Promotion, Ministry of Health and Welfare of Korea. The physical activity guide for Koreans. Korea: Ministry of Health and Welfare; 2013.

10. Hargreaves M, Spriet LL. Exercise metabolism: fuels for the fire. Cold Spring Harb Perspect Med. 2018;8:a029744. doi:10.1101/cshperspect.a029744.

11. Chiu CH, Ko MC, Wu LS, Yeh DP, Kan NW, Lee PF, et al. Benefits of different intensity of aerobic exercise in modulating body composition among obese young adults: a pilot randomized controlled trial. Health Qual Life Outcomes. 2017;15:168.

12. Dimeo F, Pagonas N, Seibert F, Arndt R, Zidek W, Westhoff TH. Aerobic exercise reduces blood pressure in resistant hypertension. Hypertension. 2012;60:653-8.

13. Nystoriak MA, Bhatnagar A. Cardiovascular effects and benefits of exercise. Front Cardiovasc Med. 2018;5:135.

14. Colberg SR, Sigal RJ, Fernhall B, Regensteiner JG, Blissmer BJ, Rubin RR, et al. Exercise and type 2 diabetes: the American College of Sports Medicine and the American Diabetes Association: joint position statement. Diabetes Care. 2010;33:e147-67.

15. Egan B, Zierath JR. Exercise metabolism and the molecular regulation of skeletal muscle adaptation. Cell Metab. 2013;17:162-84.

16. Dimeo F, Bauer M, Varahram I, Proest G, Halter U. Benefits from aerobic exercise in patients with major depression: a pilot study. Br J Sports Med. 2001;35:114-7.

17. Lattari E, Budde H, Paes F, Neto GAM, Appolinario JC, Nardi AE, et al. Effects of aerobic exercise on anxiety symptoms and cortical activity in patients with panic disorder: a pilot study. Clin Pract Epidemiol Ment Health. 2018;14:11-25.

18. Shobeiri F, Masoumi SZ, Nikravesh A, Heidari Moghadam R, Karami M. The impact of aerobic exercise on quality of life in women with breast cancer: a randomized controlled trial. J Res Health Sci. 2016;16:127-32.

19. Public health weekly report. Adults physical activity in Korea. http://nih.go.kr/board.es? $\mathrm{mid}=\mathrm{a} 30501000000 \&$ bid=0031\&list_no=364487\&act=view . Accessed 23 Jun 2019.

Page 6/10 
20. Korea Health Promotion Institute. 2017 Health Promotion Statistics Year Book. 2017. https://www.khealth.or.kr/kps/publish/fileDownload? fileGubun=site\&menuld=publishMgr\&userFileName=2017\%20\%EA\%B1\%B4\%EA\%B0\%95\%ED\%86\%B5\%EA\%B3\%84\%EC\%97\%B0\%EB\%B3\%B4(\%ED\%86\%B 0162-1000-0000-0894ef347492.pdf\&titleld=10112\&fileld=1. Accessed 23 Jun 2019.

21. Konopka AR, Harber MP. Skeletal muscle hypertrophy after aerobic exercise training. Exerc Sport Sci Rev. 2014;42:53-61.

22. Pikosky MA, Gaine PC, Martin WF, Grabarz KC, Ferrando AA, Wolfe RR, et al. Aerobic exercise training increases skeletal muscle protein turnover in healthy adults at rest. J Nutr. 2006;136:379-83.

23. Vital Statistics Division, Statistics Korea, Shin HY, Lee JY, Kim JE, Lee S, Youn H, et al. Cause-of-death statistics in 2016 in the Republic of Korea. J Korean Med Assoc. 2018;61:573-84.

24. Korean Society Hypertension (KSH), Hypertension Epidemiology Research Working Group, Kim HC, Cho MC. Korea hypertension fact sheet 2018. Clin Hypertens. 2018;24:13.

25. Munshi MN, Florez H, Huang ES, Kalyani RR, Mupanomunda M, Pandya N, et al. Management of diabetes in long-term care and skilled nursing facilities: a position statement of the American Diabetes Association. Diabetes Care. 2016;39:308-18.

26. Crane JD, Macneil LG, Tarnopolsky MA. Long-term aerobic exercise is associated with greater muscle strength throughout the life span. J Gerontol A Biol Sci Med Sci. 2013;68:631-8.

27. Al-Sharif FA, Al-Jiffri OH, El-Kader SM, Ashmawy EM. Impact of mild versus moderate intensity aerobic walking exercise training on markers of bone metabolism and hand grip strength in moderate hemophilic A patients. Afr Health Sci. 2014;14:11-6.

28. Al-Shreef FM, Al-Jiffri OH, Abd El-Kader SM. Bone metabolism and hand grip strength response to aerobic versus resistance exercise training in noninsulin dependent diabetic patients. Afr Health Sci. 2015;15:896-901.

29. Soyupek F, Bolukbasi N, Yorgancioglu ZR, Gokoglu F. The effect of aerobic exercise on hand strength and dexterity of patients with coronary artery disease. Turk J Phys Med Rehab. 2006;52:72-5.

30. Kim CR, Jeon YJ, Kim MC, Jeong T, Koo WR. Reference values for hand grip strength in the South Korean population. PLoS One. 2018;13:e0195485.

31. Phillips T, Leeuwenburgh C. Muscle fiber specific apoptosis and TNF-alpha signaling in sarcopenia are attenuated by life-long calorie restriction. FASEB J. 2005;19:668-70.

32. Chung HY, Cesari M, Anton S, Marzetti E, Giovannini S, Seo AY, et al. Molecular inflammation: underpinnings of aging and age-related diseases. Ageing Res Rev. 2009;8:18-30.

33. Janssen I, Ross R. Linking age-related changes in skeletal muscle mass and composition with metabolism and disease. J Nutr Health Aging. 2005;9:40819.

34. Ergen N, Kurdak H, Erdogan S, Mete UO, Kaya M, Dikmen N, et al. The effects of aerobic exercise on skeletal muscle metabolism, morphology and in situ endurance in diabetic rats. J Sports Sci Med. 2005;4:472-81.

35. Wallymahmed ME, Morgan C, Gill GV, MacFarlane IA. Aerobic fitness and hand grip strength in type 1 diabetes: relationship to glycaemic control and body composition. Diabet Med. 2007;24:1296-9.

36. Distefano G, Goodpaster BH. Effects of exercise and aging on skeletal muscle. Cold Spring Harb Perspect Med. 2018;8:a029785. doi:10.1101/cshperspect.a029785.

37. Fernandes T, Roque FR, Magalhães FC, do Carmo EC, de Oliveira EM. Aerobic exercise training corrects capillary rarefaction and alterations in proportions of the muscle fibers types in spontaneously hypertensive rats. Rev Bras Med Esporte. 2012;18:267-72.

\section{Tables}

Table 1 Characteristics of the study population 


\begin{tabular}{|c|c|c|c|c|c|c|}
\hline \multirow[t]{3}{*}{ Characteristic } & \multicolumn{2}{|c|}{ Total } & \multicolumn{2}{|c|}{ Men } & \multicolumn{2}{|c|}{ Women } \\
\hline & LMS & NMS & LMS & NMS & LMS & NMS \\
\hline & $(n=2,466)$ & $(n=17,184)$ & $(\mathrm{n}=1,059)$ & $(\mathrm{n}=7,617)$ & $(n=1,407)$ & $(\mathrm{n}=9,567)$ \\
\hline Age (years), mean (SD) & $65.0(16.5)^{*}$ & $48.6(15.7)^{*}$ & $66.2(16.1)^{*}$ & $48.7(15.8)^{*}$ & $64.0(16.8)^{*}$ & $48.5(15.7)^{*}$ \\
\hline \multicolumn{7}{|l|}{ Educational level, n (\%) } \\
\hline Elementary school or lower & $1289(52.6)^{*}$ & $2807(16.4)^{*}$ & $438(41.6)^{*}$ & $866(11.4)^{*}$ & $851(60.9)^{*}$ & $1941(20.3)^{*}$ \\
\hline Middle school & $285(11.6)^{*}$ & $1726(10.1)^{*}$ & $156(14.8)^{*}$ & $779(10.2)^{*}$ & $159(9.2)^{*}$ & $947(9.9)^{*}$ \\
\hline High school & $476(19.4)^{*}$ & $5944(34.6)^{*}$ & $265(25.2)^{*}$ & $2729(35.9)^{*}$ & $211(15.1)^{*}$ & $3215(33.7)^{*}$ \\
\hline College or over & $399(16.3)^{*}$ & $6681(38.9)^{*}$ & $193(18.3)^{*}$ & $3233(42.5)^{*}$ & $206(14.7)^{*}$ & $3448(36.1)^{*}$ \\
\hline \multicolumn{7}{|l|}{ Marital status, n (\%) } \\
\hline Married & $2225(90.2)^{*}$ & $14110(82.1)^{*}$ & $938(88.6)^{*}$ & $5982(78.5)^{*}$ & $1287(91.5)^{*}$ & $8128(85.0)^{*}$ \\
\hline Unmarried & $241(9.8)^{*}$ & $3074(17.9)^{*}$ & $121(11.4)^{*}$ & $1635(21.5)^{*}$ & $120(8.5)^{*}$ & $1439(15.0)^{*}$ \\
\hline \multicolumn{7}{|l|}{ Household income level, n (\%) } \\
\hline Low & $761(31.0)^{*}$ & $3913(22.8)^{*}$ & $336(31.9)^{*}$ & $1724(22.7)^{*}$ & $425(30.4)^{*}$ & $2189(23.0)^{*}$ \\
\hline Lower middle & $612(24.9)^{*}$ & $4312(25.2)^{*}$ & $263(25.0)^{*}$ & $1910(25.1)^{*}$ & $349(24.9)^{*}$ & $2402(25.2)^{*}$ \\
\hline Upper middle & $546(22.3)^{*}$ & $4416(25.8)^{*}$ & $234(22.2)^{*}$ & $1946(25.6)^{*}$ & $312(22.3)^{*}$ & $2470(25.9)^{*}$ \\
\hline High & $534(21.8)^{*}$ & $4496(26.2)^{*}$ & $220(20.9)^{*}$ & $2022(26.6)^{*}$ & $314(22.4)^{*}$ & $2474(25.9)^{*}$ \\
\hline Weight (kg), mean (SD) & $58.9(10.4)^{*}$ & $64.3(12.2)^{*}$ & $62.7(10.6)^{*}$ & $71.8(11.3)^{*}$ & 55.0) 9.0$)^{*}$ & $58.4(9.2)^{*}$ \\
\hline Height (cm), mean (SD) & $157.3(9.0)^{*}$ & $163.7(9.0)^{*}$ & $164.4(6.6)^{*}$ & $170.9(6.3)^{*}$ & $152.0(6.6)^{*}$ & $157.9(6.2)^{*}$ \\
\hline BMI $\left(\mathrm{kg} / \mathrm{m}^{2}\right)$, mean (SD) & $23.5(3.4)^{*}$ & $23.9(3.5)^{*}$ & $23.1(3.3)^{*}$ & $24.5(3.2)^{*}$ & $23.8(3.5)^{*}$ & $23.4(3.6)^{*}$ \\
\hline \multicolumn{7}{|l|}{ Smoking, n (\%) } \\
\hline Never & $1538(63.4)^{*}$ & $10272(60.1)^{*}$ & $252(24.2)^{*}$ & $1783(23.5)^{*}$ & $1286(93.0)^{*}$ & $8489(89.2)^{*}$ \\
\hline Past & $590(24.3)^{*}$ & $3547(20.8)^{*}$ & $531(51.0)^{*}$ & $2986(37.1)^{*}$ & $59(4.3)^{*}$ & $561(5.9)^{*}$ \\
\hline Current & $297(12.2)^{*}$ & $3273(19.1)^{*}$ & $259(24.9)^{*}$ & $2808(37.1)^{*}$ & $38(2.7)^{*}$ & $465(4.9)^{*}$ \\
\hline Heavy alcohol drinking, n (\%) & $121(4.9)^{*}$ & $2039(11.9)^{*}$ & $93(8.8)^{*}$ & $1531(20.1)^{*}$ & $28(2.0)^{*}$ & $508(5.3)^{*}$ \\
\hline Hypertension, n (\%) & $1194(48.5)^{*}$ & $4865(28.4)^{*}$ & $501(17.4)^{*}$ & $2532(33.3)^{*}$ & $693(49.3)^{*}$ & $2333(24.4)^{*}$ \\
\hline Diabetes, n (\%) & $475(22.3)^{*}$ & $1671(10.3)^{*}$ & $226(24.4)^{*}$ & $918(12.7)^{*}$ & $249(20.7)^{*}$ & $753(8.4)^{*}$ \\
\hline $\mathrm{FEV}_{1}(\%)$, mean (SD) & $90.33(16.3)^{*}$ & $92.24(13.2)^{*}$ & $86.6(17.5)^{*}$ & $90.0(13.3)^{*}$ & $93.3(14.6)^{*}$ & $94.0(12.8)^{*}$ \\
\hline \multicolumn{7}{|l|}{ Physical activity, n (\%) } \\
\hline$<600$ MET-min/week & $1678(68.0)^{*}$ & $8750(50.9)^{*}$ & $676(63.8)^{*}$ & $3601(47.3)^{*}$ & $1002(71.2)^{*}$ & $5149(53.8)^{*}$ \\
\hline$\geq 600 \mathrm{MET}-\mathrm{min} /$ week & $788(32.0)^{*}$ & $8434(49.1)^{*}$ & $383(36.2)^{*}$ & $4016(52.7)^{*}$ & $405(28.8)^{*}$ & $4418(46.2)^{*}$ \\
\hline Right HGS (kg), mean (SD) & $18.1(6.2)^{*}$ & $31.1(9.5)^{*}$ & $24.1(5.1)^{*}$ & $38.3(6.5)^{*}$ & $13.7(2.4)^{*}$ & $24.0(4.1)^{*}$ \\
\hline Left HGS (kg), mean (SD) & $18.5(6.7)^{*}$ & $29.6(9.4)^{*}$ & $24.7(5.1)^{*}$ & $38.3(6.5)^{*}$ & $13.9(3.0)^{*}$ & $22.7(4.0)^{*}$ \\
\hline Dominant HGS (kg), mean (SD) & $18.1(6.1)^{*}$ & $31.2(9.6)^{*}$ & $24.1(4.2)^{*}$ & $40.1(6.5)^{*}$ & $13.7(2.4)^{*}$ & $24.0(4.1)^{*}$ \\
\hline
\end{tabular}

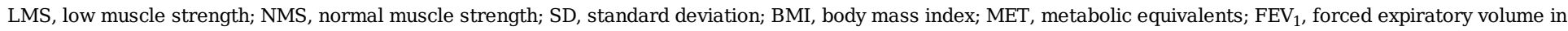

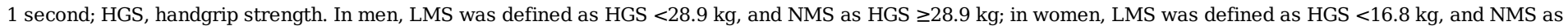
HGS $\geq 16.8 \mathrm{~kg} . P$-values: chi-square test or $t$-test, ${ }^{*} p<0.001$. 
Table 2 Odds ratios for handgrip strength in total participants and participants with chronic disease

\begin{tabular}{|c|c|c|c|}
\hline Subgroups & Model 1 [OR (95\% CI)] & Model 2 [OR (95\% CI)] & Model 3 [OR (95\% CI)] \\
\hline \multicolumn{4}{|l|}{ Total } \\
\hline$<600$ MET-min/week & $2.053(1.876-2.245)^{*}$ & $1.448(1.315-1.594)^{*}$ & $1.415(1.187-1.688)^{*}$ \\
\hline$\geq 600$ MET-min/week (ref.) & 1 & 1 & 1 \\
\hline \multicolumn{4}{|l|}{$\mathrm{Men}^{\mathrm{a}}$} \\
\hline <600 MET-min/week & $1.968(1.723-2.249)^{*}$ & $1.451(1.256-1.676)^{*}$ & $1.511(1.162-1.965)^{*}$ \\
\hline 2600 MET-min/week (ref.) & 1 & 1 & 1 \\
\hline \multicolumn{4}{|l|}{ Women $^{\mathrm{a}}$} \\
\hline <600 MET-min/week & $2.123(1.879-2.399)^{*}$ & $1.446(1.269-1.647)^{*}$ & $1.372(1.079-1.745)^{*}$ \\
\hline 2600 MET-min/week (ref.) & 1 & 1 & 1 \\
\hline \multicolumn{4}{|l|}{ Total of hypertension } \\
\hline <600 MET-min/week & $2.267(1.964-2.617)^{*}$ & $1.610(1.373-1.888)^{*}$ & $1.799(1.376-2.352)^{*}$ \\
\hline$\geq 600$ MET-min/week (ref.) & 1 & 1 & 1 \\
\hline \multicolumn{4}{|l|}{$\mathrm{Men}^{\mathrm{a}}$} \\
\hline$<600$ MET-min/week & $1.879(1.535-2.299)^{*}$ & $1.478(1.176-1.856)^{*}$ & $1.910(1.306-2.794)^{*}$ \\
\hline Z600 MET-min/week (ref.) & 1 & 1 & 1 \\
\hline \multicolumn{4}{|l|}{ Women $^{\mathrm{a}}$} \\
\hline$<600$ MET-min/week & $2.529(2.052-3.117)^{*}$ & $1.754(1.399-2.200)^{*}$ & $1.796(1.218-2.648)^{*}$ \\
\hline 2600 MET-min/week (ref.) & 1 & 1 & 1 \\
\hline \multicolumn{4}{|l|}{ Total of diabetes } \\
\hline <600 MET-min/week & $2.344(1.858-2.957)^{*}$ & $1.793(1.396-2.303)^{*}$ & $1.811(1.208-2.715)^{*}$ \\
\hline 2600 MET-min/week (ref.) & 1 & 1 & 1 \\
\hline \multicolumn{4}{|l|}{$\mathrm{Men}^{\mathrm{a}}$} \\
\hline <600 MET-min/week & $1.958(1.437-2.667)^{*}$ & $1.679(1.201-2.348)^{*}$ & $2.032(1.155-3.574)^{*}$ \\
\hline 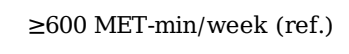 & 1 & 1 & 1 \\
\hline \multicolumn{4}{|l|}{ Women $^{\mathrm{a}}$} \\
\hline <600 MET-min/week & $2.787(1.938-4.009)^{*}$ & $2.001(1.364-2.937)^{*}$ & $1.703(0.935-3.104)$ \\
\hline 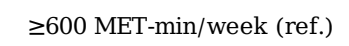 & 1 & 1 & 1 \\
\hline
\end{tabular}

BMI, body mass index; MET, metabolic equivalent. Odds ratios (OR) with adjustments using logistic regression models. Model 1: unadjusted. Model 2: adjusted for age, sex, and BMI. Model 3: adjusted for age, sex, BMI, educational level, marital status, household income level, smoking, heavy alcohol drinking, hypertension, diabetes, and $\mathrm{FEV}_{1}$. ${ }^{\mathrm{a}}$ Not adjusted for sex. ${ }^{*} p<0.05$.

\section{Figure Legend}

\section{Figure Legend}

\section{Fig. 1}

Flow diagram showing the number of participants who were excluded and the number of participants whose data were analyzed. KNHANES: Korean National Health and Nutrition Examination Survey 


\section{Figures}

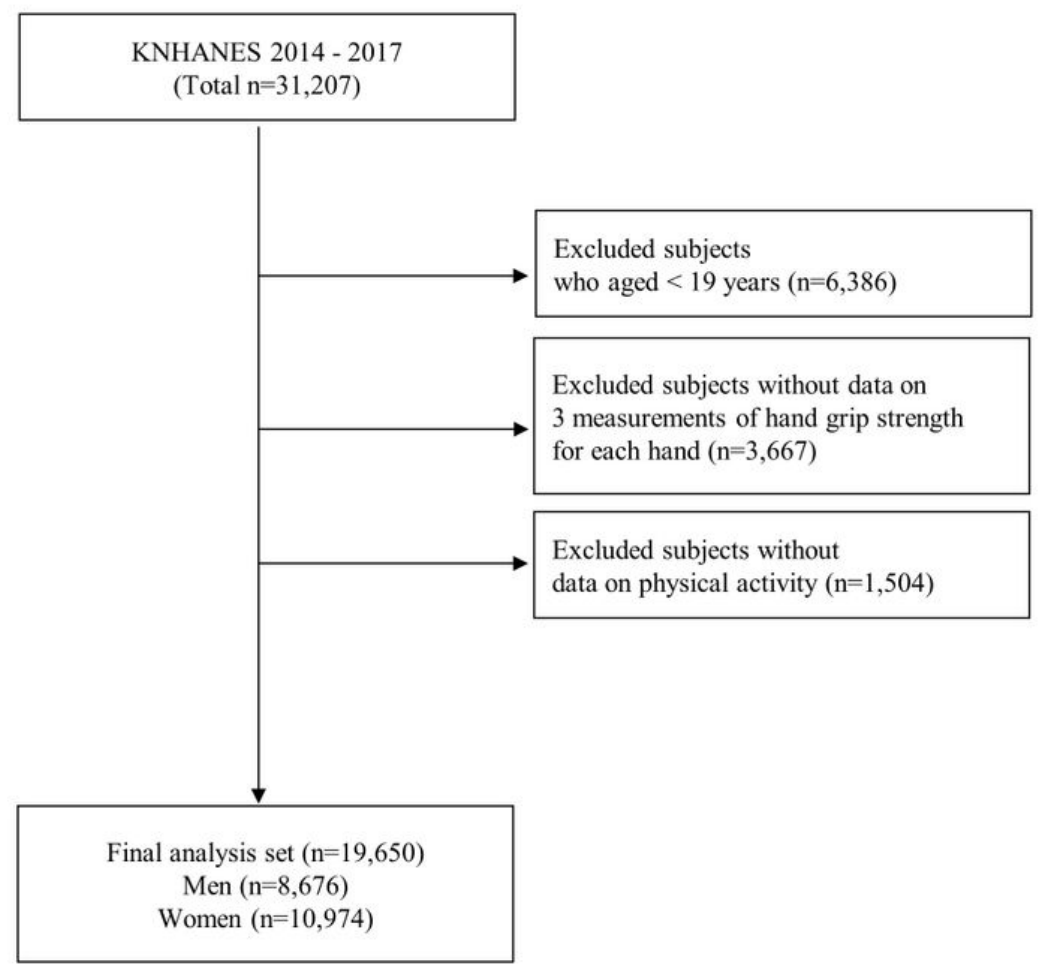

\section{Figure 1}

Flow diagram showing the number of participants who were excluded and the number of participants whose data were analyzed. KNHANES: Korean National Health and Nutrition Examination Survey 